\title{
Testing Brans-Dicke gravity with screening by scalar gravitational wave memory
}

\author{
Kazuya Koyama® \\ Institute of Cosmology \& Gravitation, University of Portsmouth, \\ Portsmouth, Hampshire PO1 3FX, United Kingdom
}

(Received 15 May 2020; accepted 28 June 2020; published 8 July 2020)

\begin{abstract}
The Brans-Dicke theory of gravity is one of the oldest ideas to extend general relativity by introducing a nonminimal coupling between the scalar field and gravity. The Solar System tests put tight constraints on the theory. In order to evade these constraints, various screening mechanisms have been proposed. These screening mechanisms allow the scalar field to couple to matter as strongly as gravity in low density environments while suppressing it in the Solar System. The Vainshtein mechanism, which is found in various modified gravity models such as massive gravity, braneworld models and scalar tensor theories, suppresses the scalar field efficiently in the vicinity of a massive object. This makes it difficult to test these theories from gravitational wave observations. We point out that the recently found scalar gravitational wave memory effect, which is caused by a permanent change in spacetime geometry due to the collapse of a star to a back hole can be significantly enhanced in the Brans-Dicke theory of gravity with the Vainshtein mechanism. This provides a possibility to detect scalar gravitational waves by a network of three or more gravitational wave detectors.
\end{abstract}

DOI: 10.1103/PhysRevD.102.021502

\section{INTRODUCTION}

The detection of gravitational waves has provided new possibilities to test the theory of gravity [1,2]. Many modified gravity models introduce additional degrees of freedom in gravity and the detection of additional polarizations of gravitational waves will give a smoking gun for deviations from general relativity (GR) [3]. In the scalar tensor theories, we expect the presence of the scalar gravitational waves [4-6].

However, scalar tensor theories are highly constrained by the Solar System measurements [7]. These constraints limit the strength of the coupling between the scalar field and matter. This makes it difficult to test these theories beyond the Solar System. Various screening mechanisms have been proposed to evade the Solar System constraints without suppressing the coupling $[8,9]$. There are two main screening mechanisms. One is to suppress the scalar field gradient at the vicinity of an object. The representative example is the Vainshtein mechanism that can be found in various modified gravity models such as massive gravity, higherdimensional braneworld model as well as scalar tensor theories [10] (see [11] for a review). The other example is the chameleon mechanism [12,13] (see a review [14]). In this case, the scalar field mass changes depending on environments, suppressing the coupling between the scalar field and matter. The effectiveness of screening is determined by the spatial curvature of the object in the Vainshtein mechanism and the gravitational potential of the object in the chameleon mechanism. These screening mechanisms have distinct effects on structures in the Universe [15]. Given the nature of these screening mechanisms to suppress deviations from GR in an environment with a large curvature/gravitational potential, we expect that deviations from GR will be suppressed further in a system such as binary black holes or neutron stars. This makes it difficult to test these models with gravitational wave observations [16].

Recently, a novel way to test scalar tensor theories was proposed using the gravitational wave memory [17]. The gravitational wave memory is a permanent change in spacetime geometry [18]. In GR, this is caused by a burst event, which creates a jump in the transverse-traceless part of the space-time metric $[19,20]$. In scalar tensor theories, the scalar mode of metric perturbations leads to a new scalar gravitational wave memory [17]. Imagine a star that collapses to a black hole. Initially the scalar field is supported by the star. After the collapse, due to the nohair theorem [21], the black hole does not support the scalar field. This causes a permanent change in the scalar component of metric perturbations outside the star, leading to the gravitational memory. It was shown that using a network of three or more detectors, it is possible to separate the scalar component of the gravitational waves [22] as different gravitational wave detectors on various locations have distinct responses to the different polarizations. Using a network of LIGO-Hanford, LIGO-Livingston [23], Virgo [24] and KAGRA [25], it is possible to detect the scalar gravitational memory from a collapse of a $10 M_{\odot}$ star at the distance of $10 \mathrm{kpc}$ can be detected in the Brans-Dicke 
theory by the second generation of gravitational wave detector network even imposing the Solar System constraint [17]. The memory effect dominates the scalar stochastic gravitational wave background below $100 \mathrm{~Hz}$ [26].

The no-hair theorem applies to the scalar tensor theories [27] as well as shift-symmetric Galileon theories [28], which accommodate the Vainshtein mechanism [29]. A number of ways out exist for the black hole no-hair theorem (see [30,31] for reviews). For example, hairy black hole solutions can be found in Galileon theories if the scalar field is allowed to acquire time-dependence [32]. Hairy black holes can be found also in general relativity coupled with various matter fields as well as in a theory with a linear coupling of the scalar with the Gauss-Bonnet invariant [33]. We will come back to this point in the discussion.

In this paper, we show that the scalar memory effect provides a powerful way to test Brans-Dicke gravity with the Vainshtein mechanism. The Vainshtein mechanism is very difficult to test due to its very efficient suppression of the scalar force. The scalar memory effect will provide constraints that cannot be reached by the Solar System tests as well as astrophysical tests [34] and offer a possibility to discover scalar gravitational waves with high signal-tonoise ratio from a nearby gravitational collapse with a network of the second generation of gravitational wave detectors. We will also show that the scalar memory effect is suppressed in the chameleon mechanism.

\section{BRANS-DICKE THEORY WITH VAINSHTEIN MECHANISM}

As a concrete example, we consider the model described by the following action [35]

$$
\int d^{4} x \sqrt{-g}\left[\phi R-\frac{\omega}{\phi}(\partial \phi)^{2}+f(\phi) \square \phi(\partial \phi)^{2}+\mathcal{L}_{m}\right],
$$

where $\omega$ is the Brans-Dicke parameter, $(\partial \phi)^{2}=\partial^{\alpha} \phi \partial_{\alpha} \phi$ and $\mathcal{L}_{m}$ is the matter Lagrangian. The cubic interaction is the unique form of interactions at this order that keeps the field equation for $\phi$ of second-order [29].

We expand the metric and the scalar field as $g_{\mu \nu}=$ $\eta_{\mu \nu}+h_{\mu \nu}$ and $\phi=\phi_{0}(1+\varphi)$. By keeping nonlinear terms in the second derivative of $\varphi$, which are relevant to the Vainshtein mechanism, the equations of motion become

$$
\begin{aligned}
\delta G_{\mu \nu}\left(h_{\mu \nu}\right)= & \left(\partial_{\mu} \partial_{\nu} \varphi-\eta_{\mu \nu} \square \varphi\right)+\frac{1}{2 \phi_{0}} \delta T_{\mu \nu}, \\
(3+2 \omega) \square \varphi= & -2 f\left(\phi_{0}\right) \phi_{0}^{2}\left[\left(\partial_{\mu} \partial_{\nu} \varphi\right)\left(\partial^{\mu} \partial^{\nu} \varphi\right)-(\square \varphi)^{2}\right] \\
& +\frac{\delta T_{\mu}^{\mu}}{2 \phi_{0}},
\end{aligned}
$$

where $\delta G_{\mu \nu}\left(h_{\mu \nu}\right)$ is the linearized Einstein tensor and $\delta T_{\mu, \nu}$ is the linearized energy momentum tensor. Introducing
$H_{\mu \nu}=h_{\mu \nu}-\eta_{\mu \nu} \varphi$, we can diagonalize the equations for $H_{\mu \nu}$ and $\varphi: \delta G_{\mu \nu}\left(H_{\mu \nu}\right)=\delta T_{\mu \nu} / 2 \phi_{0}$.

In the static limit, the scalar field equation becomes

$$
\nabla^{2} \varphi+r_{c}^{2}\left[\left(\partial_{l} \partial_{j} \varphi\right)\left(\partial^{i} \partial^{j} \varphi\right)-\left(\partial^{2} \varphi\right)^{2}\right]=-8 \pi G \alpha^{2} \rho,
$$

where we defined

$\phi_{0}=(16 \pi G)^{-1}, \quad \alpha=(2 \omega+3)^{-\frac{1}{2}}, \quad r_{c}^{2}=\frac{2 f\left(\phi_{0}\right) \phi_{0}^{2}}{3+2 \omega}$.

The parameter $\alpha$ controls the coupling between the scalar field and matter and $r_{c}$ controls the efficiency of the Vainshtein mechanism. The spherically symmetric solution is given by [36]

$$
\begin{aligned}
\varphi(r) & =\frac{r_{V}^{2}}{4 r_{c}^{2}} g\left(\frac{r}{r_{V}}\right), \\
g(x) & =-\frac{x^{2}}{2}\left[-1+{ }_{2} F_{1}\left(-\frac{2}{3},-\frac{1}{2}, \frac{1}{3},-x^{-3}\right)\right],
\end{aligned}
$$

where

$$
r_{V}=\left(16 \alpha^{2} r_{c}^{2} G M\right)^{1 / 3}
$$

is the Vainshtein radius and we imposed the condition that $\varphi(r) \rightarrow 0$ at $r \rightarrow \infty$. Figure 1 shows the profile of the scalar field. On larger scales $r>r_{V}$, the solution approaches the linear solution

$$
\varphi=\frac{2 \alpha^{2} G M}{r}
$$

On the other hand, the scalar field is highly suppressed at $r \ll r_{V}$, realizing the Vainshtein mechanism. The important point is that the Vainshtein mechanism is not a screening mechanism in the standard sense. Normally the screening mechanism is a mechanism to reduce a charge. In the case

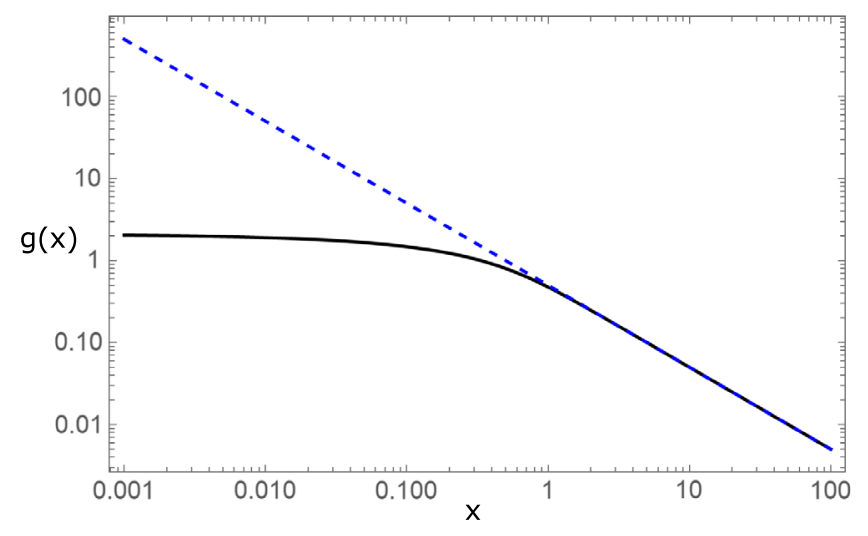

FIG. 1. The function $g(x)$ in Eq. (5). The dotted line shows the linear solution 2/ $x$ for $x \gg 1$. Below the Vainshtein radius $x<1$ the scalar field is suppressed due to the nonlinear term. 
of the Vainshtein mechanism, the scalar charge is given by $2 \alpha^{2} M$ and this is not suppressed. The Vainshtein mechanism suppresses the scalar field gradient inside the Vainshtein radius due to the nonlinear derivative interaction. Thanks to this feature, $\alpha$ can be $\mathrm{O}(1)$, i.e., the scalar force is as strong as gravity.

\section{SCALAR GRAVITATIONAL WAVE MEMORY}

Now, we consider the gravitational collapse of a star that forms a black hole. The no hair theorem of black holes states that black holes only support a trivial scalar field $\phi=\phi_{0}=$ const. In fact, $\phi=$ const. is a solution without matter in our theory. Assuming that the no-hair theorem holds and the scalar hair is lost, the scalar field solution becomes $\phi=\phi_{0}$ from $\phi=\phi_{0}+\varphi(r)$. This causes a permanent change in the scalar component of the metric perturbation

$$
\Delta h_{i j}^{S}=\varphi(r) e_{i j}^{o},
$$

where $e_{i j}^{o}$ is the scalar polarization tensor. As long as the Solar System is located beyond the Vainshtein radius of the collapsing star, the scalar field solution is given by Eq. (7). Figure 2 shows the Vainshtein radius for a star with $M=$ $1 M_{\odot}$ and $10 M_{\odot}$ with $\alpha=1$. For $r_{c}=1000 \mathrm{Mpc}$, the Vainshtein radius for the Sun is $0.1 \mathrm{kpc}$ and the Solar system constraint is well satisfied. On the other hand, for $M=10 M_{\odot}$, the Vainshtein radius is still $0.2 \mathrm{kpc}$ and the linear solution can be used safely in the Solar System. In order for the Solar System to be inside the Vainshtein radius of the star with $M=10 M_{\odot}$ at the distance of $10 \mathrm{kpc}$ from the Sun, $r_{c}$ needs to be $3.6 \times 10^{5} \mathrm{Mpc}$, which is well beyond the current horizon scale of the universe. Thus, for a reasonable choice of the parameters, we can approximate $\varphi(r)$ on Earth by the linear solution. Figure 3 shows the

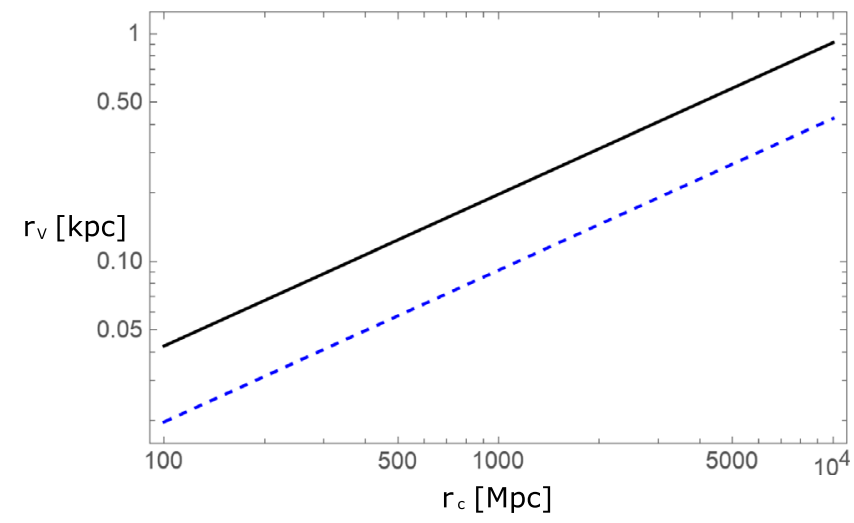

FIG. 2. The Vainshtein radius $r_{v}$ in the unit of kpc as a function of $r c$ in the unit of Mpc for $\alpha=1$. The cosmological horizon scale today is $2998 h^{-1} \mathrm{Mpc}$ where $h \sim 0.7$. The solid line is for a star with $M=10 M_{\odot}$ and the dashed line is for a solar mass star.

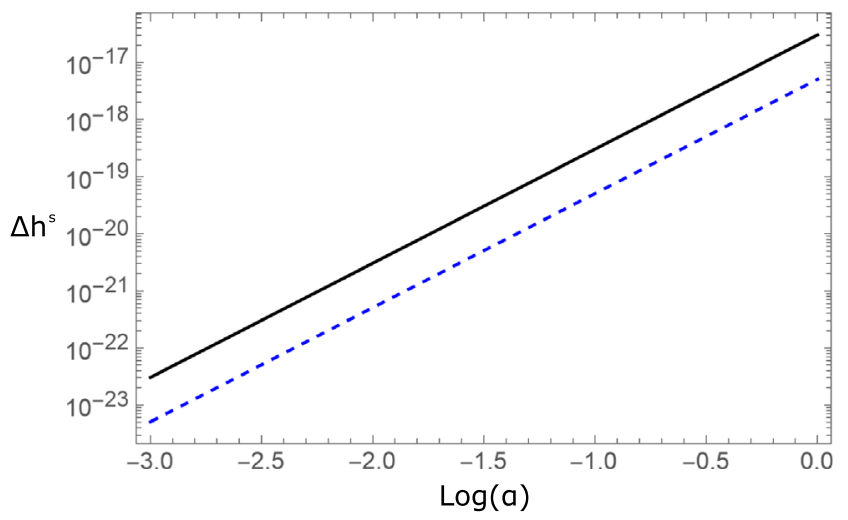

FIG. 3. The change of the scalar component of metric perturbations given by Eq. change as a function of $\alpha$. The solid line is for a star with $M=10 M_{\odot}$ at $10 \mathrm{kpc}$ and the dashed line is for a star with $M=10 M_{\odot}$ at $60 \mathrm{kpc}$.

change of the scalar component of the metric perturbation as a function of $\alpha$ assuming the linear solution.

The scalar gravitational wave memory has a frequency dependence of $h(f) \sim 1 / f$ [26]. The maximized signal to noise ratio (SNR) for the detection of the scalar memory is given by [17]

$$
\rho=\mathcal{F}_{N}^{1 / 2} \frac{2 \alpha^{2} G M}{\pi r}\left[\int_{0}^{f_{c}} d f \frac{1}{f^{2} S_{n}(f)}\right]
$$

where $S_{n}(f)$ is the noise spectral density that we assume all the detectors approximately have and $f_{c}$ is the cutoff frequency of memory determined by the time scale of the collapse. For a star with $M=10 M_{\odot}$ and $R=100 M_{\odot}$, this is estimated as $f_{c}=500 \mathrm{~Hz}$. $\mathcal{F}_{N}$ is the $\mathrm{N}$-detector effective angular pattern function and it is angular position dependent [22]. The angular averaged value for 3 (4) detectors is $F_{3}(4)=0.087(0.240)$ and the peak value is $F_{3}(4)=$ $0.511(0.240)$. We use the noise spectral density given in Ref. [37] for the second and third generation detectors. Note that the detector configurations for the third generation detector considered in Ref. [37] are outdated but we used the same specifications that were used in [17] to make a comparison easier. The scalar overlap reduction function for the Einstein telescope was computed in Ref. [26].

Figure 4 shows the discovery curve defined as $\mathrm{SNR}=$ 10 with $M=10 M_{\odot}$ as a function of the distance to the star. As shown in [17], the second generation detectors could discover the scalar gravitational memory if the star is $10 \mathrm{kpc}$ away even in the Brans-Dicke gravity with no screening, for which the Solar System constraint imposes $\alpha<10^{-2.45}$. If the distance is larger than $10 \mathrm{kpc}$, the third generation detectors would be required. On the other hand, the Vainshtein mechanism removes the limit on $\alpha$ from the Solar System constraint. The coupling can be as large as $\alpha \sim O(1)$. In this case, the second generation detectors 


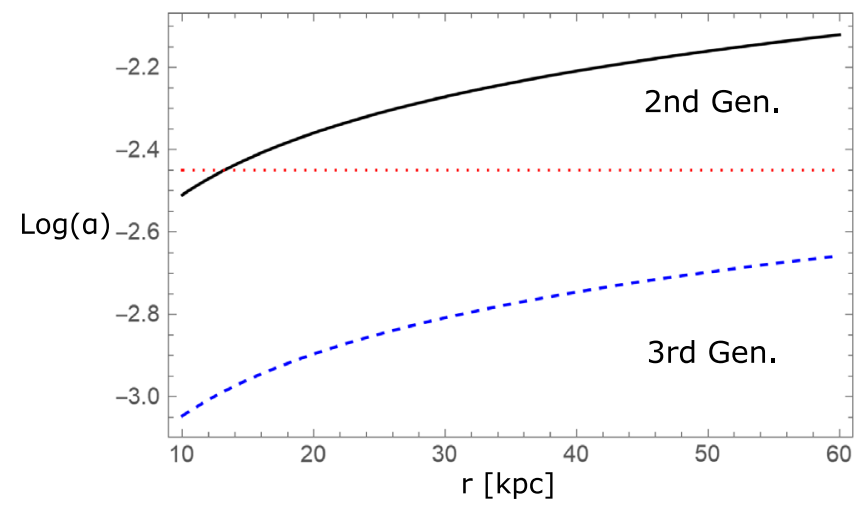

FIG. 4. The discovery threshold for the scalar gravitational memory from a collapsing star with $M=10 M_{\odot}$ as a function of the distance from Earth in the unit of kpc. If $\alpha$ is above the curve, the signal will be detected with $\mathrm{SNR}=10$. The solid (dashed) curve is for the 2nd (3rd) generation of detector network. The dotted line shows the Cassini bound (upper bound) in the Solar System as a reference. We emphasize that this bound does not apply to the theory with the Vainshtein mechanism and $\alpha$ can be $O(1)$.

would discover the scalar gravitational wave memory with $S N R>10^{5}$ as shown in Fig. 5.

\section{DISCUSSIONS}

We assumed that the Vainshtein mechanism of the Sun does not affect the scalar field profile generated by the distant star. This is a valid approximation thanks to the Galileon symmetry of Eq. (3) [38]. Since the equation contains only the second derivatives, it is possible to add a constant gradient. Since the scalar field gradient generated

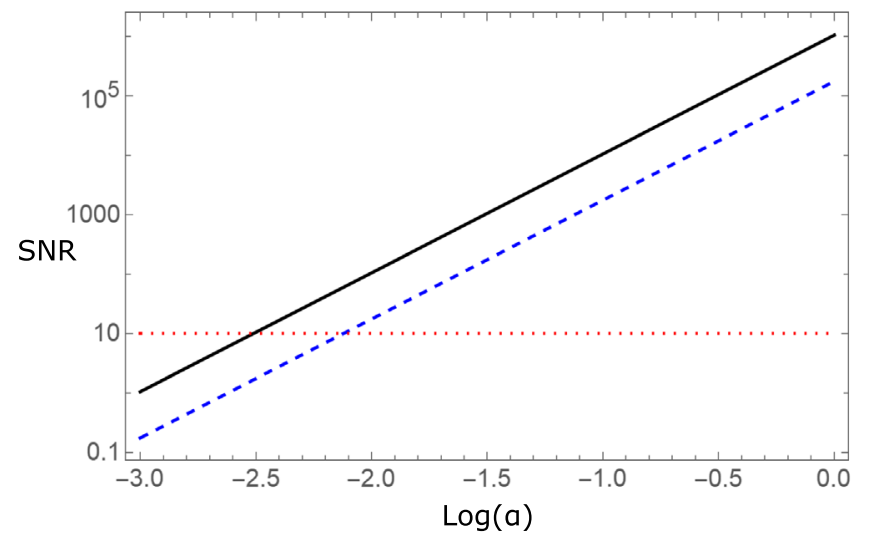

FIG. 5. The SNR for the detection of the scalar gravitational wave memory in Brans-Dicke gravity with the Vainshtein mechanism from a collapsing star with $M=10 M_{\odot}$ at the distance of $10 \mathrm{kpc}$ (solid line) and $60 \mathrm{kpc}$ (dashed line) with the 2 nd generation of detectors. This is valid as long as the Vainshtein radius of the star is less than $10(60) \mathrm{kpc}$. The vertical dotted line shows SNR $=10$ used in Fig. 4. by the distant star can be approximated as constant at the vicinity of the Sun, the solution can be written as

$$
\nabla \phi(r)=\nabla \phi_{\text {Sun }}(r)+\nabla \phi_{\text {star }} .
$$

This is not the case if the Sun is inside the Vainshtein radius of the star [39], but, in this case, the scalar field generated by the star is also suppressed.

In this paper we used a weak field limit solution. The Vainshtein mechanism works also for relativistic stars $[40,41]$ and the linear solution is recovered beyond the Vainshtein radius. As discussed in the Introduction, black holes can have a scalar hair if the scalar field has a time dependence in Galileon theories [31] although it is not clear whether hairy solutions can be formed as a result of the dynamical gravitational collapse or not. Since the existence of the nontrivial scalar field for a neutron star and the absence of it for a black hole are central to the scalar gravitational memory effect, it is important to derive neutron star solutions and prove the no-hair theorem for dynamically formed black holes in the theory described by Eq. (1). This is left for future work.

It is difficult to test the Vainshtein mechanism with $r_{c}$ close to the horizon scale today. The lunar laser ranging and the observed precession of planets in the Solar System give constraints $r_{c}>$ a few hundreds Mpc [42]. A better constraint is obtained by super massive black holes at the center of a galaxy [34]. This test shares common ideas behind the scalar gravitational wave memory test based on the observation made in [38]. Due to the no-hair theorem of black holes, if part of a galaxy's motion is due to an external scalar field sourced by a cluster where the galaxy is located, the supermassive black hole that lies at its center does not feel this. On the other hand, for a galaxy located within a cluster, the scalar field sourced by the cluster behaves as a constant-gradient field as in Eq. (10) and the resident stars and dark matter of the galaxy respond to this clustersourced scalar field. Therefore the black hole lags behind as the galaxy moves. The absence of this lag put constraint on $r_{c}$. Although it is possible to get a better constraint than the Solar System test, for $\alpha=O(1)$, the constraint remains to be $r_{c}>$ several hundreds Mpc. The gravitational memory effect can prove the regime where $r_{c}$ is close to or even larger than the horizon scale.

Finally we contrast the Vainshtein mechanism against another screening mechanism, the chameleon mechanism. In this model, the scalar charge is suppressed if the thin shell condition is satisfied, which is determined by the gravitational potential of the object. To satisfy the Solar System constraint, the Milky Way galaxy with the gravitational potential $10^{-5}$ needs to satisfy the thin shell condition. The collapsing star has a much larger gravitational potential and it is fully screened. Thus the scalar field sourced by the star is highly suppressed. Also the scalar field is screened on Earth. Unlike the Vainshtein mechanism, the superposition of 
scalar field gradients does not hold and if the scalar field is suppressed by Earth, then the external scalar field is also screened [43]. Thus we do not expect to see the scalar gravitational wave memory effect in theories with the chameleon mechanism.

The scalar gravitational wave memory from a collapsing star has a potential to discover the Brans-Dicke theory of gravity that is indistinguishable from GR in the Solar System. In particular, the theory that utilize the Vainshtein mechanism will give a strong signal with SNR reaching $10^{5}$ even with the second generation of gravitational wave detector network. We need some luck with this test given the expected gravitational collapse rate of 2 events per 100 years within $60 \mathrm{kpc}$ (note that this rate depends on star formation rates and other factors in the gravitational collapse [44]). However, if we detect the scalar gravitational waves together with the transverse traceless gravitational waves, this is a smoking gun of the theory beyond Einstein's theory of gravity.

\section{ACKNOWLEDGMENTS}

K. K. thanks Jeremy Sakstein for useful discussions. K. K. has received funding from the European Research Council (ERC) under the European Unions Horizon 2020 research and innovation programme (Grant Agreement No. 646702 CosTesGrav) and the UK Science and Technologies Facilities Council Grant No. ST/S000550/1.
[1] B.P. Abbott et al., Tests of General Relativity with GW150914, Phys. Rev. Lett. 116, 221101 (2016); Erratum, Phys. Rev. Lett. 121, 129902 (2018).

[2] B. P. Abbott et al., Tests of general relativity with the binary black hole signals from the LIGO-Virgo catalog GWTC-1, Phys. Rev. D 100, 104036 (2019).

[3] D. M. Eardley, D. L. Lee, A. P. Lightman, R. V. Wagoner, and C. M. Will, Gravitational-Wave Observations as a Tool for Testing Relativistic Gravity, Phys. Rev. Lett. 30, 884 (1973).

[4] M. Maggiore and A. Nicolis, Detection strategies for scalar gravitational waves with interferometers and resonant spheres, Phys. Rev. D 62, 024004 (2000).

[5] A. Nishizawa, A. Taruya, K. Hayama, S. Kawamura, and M.-A. Sakagami, Probing non-tensorial polarizations of stochastic gravitational-wave backgrounds with groundbased laser interferometers, Phys. Rev. D 79, 082002 (2009).

[6] T. Callister, A. Sylvia Biscoveanu, N. Christensen, M. Isi, A. Matas, O. Minazzoli, T. Regimbau, M. Sakellariadou, J. Tasson, and E. Thrane, Polarization-based Tests of Gravity with the Stochastic Gravitational-Wave Background, Phys. Rev. X 7, 041058 (2017).

[7] C. M. Will, The confrontation between general relativity and experiment, Living Rev. Relativity 17, 4 (2014).

[8] A. Joyce, B. Jain, J. Khoury, and M. Trodden, Beyond the cosmological standard model, Phys. Rep. 568, 1 (2015).

[9] K. Koyama, Cosmological tests of modified gravity, Rep. Prog. Phys. 79, 046902 (2016).

[10] A. I. Vainshtein, To the problem of nonvanishing gravitation mass, Phys. Lett. 39B, 393 (1972).

[11] E. Babichev and C. Deffayet, An introduction to the Vainshtein mechanism, Classical Quantum Gravity 30, 184001 (2013).

[12] J. Khoury and A. Weltman, Chameleon Fields: Awaiting Surprises for Tests of Gravity in Space, Phys. Rev. Lett. 93, 171104 (2004).
[13] J. Khoury and A. Weltman, Chameleon cosmology, Phys. Rev. D 69, 044026 (2004).

[14] C. Burrage and J. Sakstein, A compendium of chameleon constraints, J. Cosmol. Astropart. Phys. 11 (2016) 045.

[15] B. Falck, K. Koyama, and G.-B. Zhao, Cosmic web and environmental dependence of screening: Vainshtein vs chameleon, J. Cosmol. Astropart. Phys. 07 (2015) 049.

[16] F. Dar, C. De Rham, J. Tate Deskins, J. T. Giblin, and A. J. Tolley, Scalar gravitational radiation from binaries: Vainshtein mechanism in time-dependent systems, Classical Quantum Gravity 36, 025008 (2019).

[17] S. M. Du and A. Nishizawa, Gravitational wave memory: A new approach to study modified gravity, Phys. Rev. D 94, 104063 (2016).

[18] V. B. Braginskii and K. S. Thorne, Gravitational-wave bursts with memory and experimental prospects, Nature (London) 327, 123 (1987).

[19] Y. B. Zel'dovich and A. G. Polnarev, Radiation of gravitational waves by a cluster of superdense stars., Sov. Astron. 18, 17 (1974).

[20] D. Christodoulou, Nonlinear Nature of Gravitation and Gravitational Wave Experiments, Phys. Rev. Lett. 67, 1486 (1991).

[21] S. W. Hawking, Black holes in general relativity, Commun. Math. Phys. 25, 152 (1972).

[22] K. Hayama and A. Nishizawa, Model-independent test of gravity with a network of ground-based gravitational-wave detectors, Phys. Rev. D 87, 062003 (2013).

[23] https://www.ligo.caltech.edu/.

[24] http://www.virgo gw.eu/.

[25] https://gwcenter.icrr.u tokyo.ac.jp/en/.

[26] S. M. Du, Scalar stochastic gravitational-wave background in Brans-Dicke theory of gravity, Phys. Rev. D 99, 044057 (2019).

[27] T. P. Sotiriou and V. Faraoni, Black Holes in Scalar-Tensor Gravity, Phys. Rev. Lett. 108, 081103 (2012).

[28] L. Hui and A. Nicolis, No-Hair Theorem for the Galileon, Phys. Rev. Lett. 110, 241104 (2013). 
[29] A. Nicolis, R. Rattazzi, and E. Trincherini, The Galileon as a local modification of gravity, Phys. Rev. D 79, 064036 (2009).

[30] C. A. R. Herdeiro and E. Radu, Asymptotically flat black holes with scalar hair: A review, Int. J. Mod. Phys. D 24, 1542014 (2015).

[31] E. Babichev, C. Charmousis, and A. Lehebel, Black holes and stars in Horndeski theory, Classical Quantum Gravity 33, 154002 (2016).

[32] E. Babichev and C. Charmousis, Dressing a black hole with a time-dependent Galileon., J. High Energy Phys. 08 (2014) 106.

[33] T. P. Sotiriou and S.-Y. Zhou, Black Hole Hair in Generalized Scalar-Tensor Gravity, Phys. Rev. Lett. 112, 251102 (2014).

[34] J. Sakstein, B. Jain, J. S. Heyl, and L. Hui, Tests of gravity theories using supermassive black holes., Astrophys. J. Lett. 844, L14 (2017).

[35] F. P. Silva and K. Koyama, Self-accelerating universe in Galileon cosmology, Phys. Rev. D 80, 121301 (2009).

[36] F. Schmidt, W. Hu, and M. Lima, Spherical collapse and the halo model in braneworld gravity, Phys. Rev. D 81, 063005 (2010).
[37] B. S. Sathyaprakash and B. F. Schutz, Physics, astrophysics and cosmology with gravitational waves, Living Rev. Relativity 12, 2 (2009).

[38] L. Hui, A. Nicolis, and C. Stubbs, Equivalence principle implications of modified gravity models, Phys. Rev. D 80, 104002 (2009).

[39] T. Hiramatsu, W. Hu, K. Koyama, and F. Schmidt, Equivalence principle violation in vainshtein screened two-body systems, Phys. Rev. D 87, 063525 (2013).

[40] J. Chagoya, K. Koyama, G. Niz, and G. Tasinato, Galileons and strong gravity, J. Cosmol. Astropart. Phys. 10 (2014) 055 .

[41] H. Ogawa, T. Kobayashi, and K. Koyama, Relativistic stars in a cubic Galileon Universe, Phys. Rev. D 101, 024026 (2020).

[42] G. Dvali, A. Gruzinov, and M. Zaldarriaga, The Accelerated universe and the moon, Phys. Rev. D 68, 024012 (2003).

[43] W. Hu, Acceleration from modified gravity: Lessons from worked examples, Nucl. Phys. B Proc. Suppl. 194, 230 (2009).

[44] K. C.B. New, Gravitational waves from gravitational collapse, Living Rev. Relativity 6, 2 (2003). 